\title{
Radiation exposure dose of fluoroscopy-guided gastrointestinal procedures: A single-center retrospective study
}

\section{(ㄷ)(1) $\odot$}

Authors

Shiro Hayashi ${ }^{1}{ }^{1,2}$, Tsutomu Nishida ${ }^{1}$, Shinji Kuriki ${ }^{1}$, Li-sa Chang ${ }^{1}$, Kazuki Aochi ${ }^{1}$, Emi Meren ${ }^{1}$, Tatsuya Sakamoto ${ }^{1}$, Ryo Tomita ${ }^{1}$, Yu Higaki ${ }^{1}$, Naoto Osugi ${ }^{1}$, Aya Sugimoto ${ }^{1}$, Kei Takahashi ${ }^{1}$, Kaori Mukai ${ }^{1}$, Kengo Matsumoto ${ }^{1}$, Dai Nakamatsu', Masahi Yamamoto ${ }^{1}$, Koji Fukui ${ }^{1}$, Mamoru Takenaka ${ }^{3}$, Makoto Hosono ${ }^{4}$, Masami Inada ${ }^{1}$

Institutions

1 Department of Gastroenterology, Toyonaka Municipal Hospital, Osaka, Japan

2 Department of Gastroenterology and Internal Medicine, Hayashi Clinic, Osaka, Japan

3 Department of Gastroenterology and Hepatology, Kindai University Faculty of Medicine, Osaka, Japan

4 Department of Radiology, Kindai University Faculty of Medicine, Osaka, Japan

submitted 20.5.2020

accepted after revision 21.9.2020

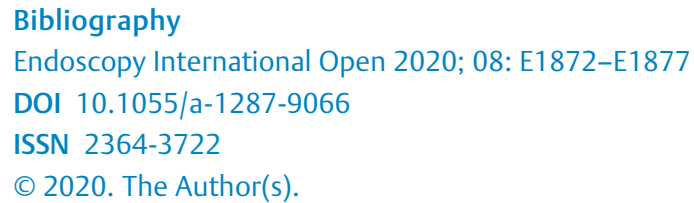

This is an open access article published by Thieme under the terms of the Creative Commons Attribution-NonDerivative-NonCommercial License, permitting copying and reproduction so long as the original work is given appropriate credit. Contents may not be used for commecial purposes, or adapted, remixed, transformed or built upon. (https://creativecommons.org/licenses/by-nc-nd/4.0/)

\section{Corresponding author}

Tsutomu Nishida, MD, PhD, Department of Gastroenterology, Toyonaka Municipal Hospital, 4-14-1 Shibahara, Toyonaka, Osaka 560-8565, Japan

Fax: +81-6-6858-3531

tnishida.gastro@gmail.com
ABSTRACT

Background and study aims Fluoroscopy-guided gastrointestinal procedures (FGPs) are increasingly common. However, the radiation exposure (RE) to patients undergoing FGPs is still unclear. We examined the actual RE of FGPs. Patients and methods This retrospective, single-center cohort study included consecutive FGPs, including endoscopic retrograde cholangiopancreatography (ERCP), interventional endoscopic ultrasound (EUS), enteral stenting, balloon-assisted enteroscopy, tube placement, endoscopic injection sclerotherapy (EIS), esophageal balloon dilatation and repositioning for sigmoid volvulus, from September 2012 to June 2019. We measured the air kerma (AK, mGy), dose area product (DAP, Gycm²), and fluoroscopy time (FT, min) for each procedure.

Results In total, 3831 patients were enrolled. Overall, 2778 ERCPs were performed. The median AK, DAP, and FT were as follows: ERCP: $109 \mathrm{mGy}, 13.3 \mathrm{Gycm}^{2}$ and $10.0 \mathrm{~min}$; self-expandable enteral stenting (SEMS): $62 \mathrm{mGy}$, $12.4 \mathrm{Gycm}^{2}$ and $10.4 \mathrm{~min}$; tube placement: $40 \mathrm{mGy}$, $13.8 \mathrm{Gycm}^{2}$ and $11.1 \mathrm{~min}$; balloon-assisted enteroscopy: $43 \mathrm{mGy}, 22.4 \mathrm{Gycm}^{2}$ and $18.2 \mathrm{~min}$; EUS cyst drainage (EUSCD): $96 \mathrm{mGy}, 18.3 \mathrm{Gycm}^{2}$ and $10.4 \mathrm{~min}$; EIS: $36 \mathrm{mGy}$, $8.1 \mathrm{Gycm}^{2}$ and $4.4 \mathrm{~min}$; esophageal balloon dilatation: $9 \mathrm{mGy}, 2.2 \mathrm{Gycm}^{2}$ and $1.8 \mathrm{~min}$; and repositioning for sigmoid volvulus: $7 \mathrm{mGy}, 4.7 \mathrm{Gycm}^{2}$ and $1.6 \mathrm{~min}$.

Conclusion This large series reporting actual RE doses of various FGPs could serve as a reference for future prospective studies.

\section{Introduction}

Recently, minimally invasive procedures for medical purposes have become more in demand. The trend of increasing radiation exposure (RE) from medical imaging has led to significant concerns about the safety of both patients and medical staff because of its adverse effects, which are represented by two major processes: tissue reactions and carcinogenesis [1-4]. The optimization of patient protection from RE from diagnostic and interventional procedures requires the application of ex-

amination-specific protocols. The International Commission on Radiological Protection (ICRP) and International Atomic Energy Agency (IAEA) introduced diagnostic reference levels (DRLs) to optimize RE from medical imaging with ionizing radiation [5]. DRLs should be managed appropriately to avoid these adverse effects. The ICRP recommends that DRLs should be used to manage patient doses during both diagnostic and interventional procedures. However, it is difficult to apply the DRL concept to interventional procedures because the RE level depends on 
the individual clinical circumstances and the complexity of the procedure.

The Japan Network for Research and Information on Medical Exposure (J-RIME) released Japan DRLs in 2015 [6, 7], which described a fluoroscopic radiation dose rate of $20 \mathrm{mGy} / \mathrm{min}$ for fluoroscopically guided interventional procedures. However, these DRLs were not for specific procedures. Based on the Japan DRLs, radiology and cardiology societies updated theirs guidelines for radiational protection [8]. Regarding the field of gastrointestinal endoscopy, various fluoroscopy-guided gastrointestinal procedures (FGPs) are rapidly increasing in popularity, including endoscopic retrograde cholangiopancreatography (ERCP), interventional endoscopic ultrasound (EUS), and enteral stenting $[9,10]$, but DRLs have not yet been established in the field of gastroenterology. The American Society for Gastrointestinal Endoscopy (ASGE) recommends applying the frequency with which fluoroscopy time and radiation dose are measured and documented as a quality indicator for ERCP [11]. The European Society of Gastrointestinal Endoscopy (ESGE) guidelines on radiation protection in digestive endoscopy recommend the establishment of DRLs for ERCP; however, they referred to a small sample size and concluded that "little progress has been made so far" [12].

The ICRP 118 announced a new threshold level for tissue reactions and decreased the annual limit of occupational exposure to the eye lens down to approximately one-seventh (an equivalent dose limit for the lens of the eye of $20 \mathrm{mSv} / \mathrm{year}$ ) of that in 2012 [13] to avoid adverse effects. According to this statement, radiology and cardiovascular societies and the ESGE have stated radiation protection measures for occupational exposure $[8,12,14]$. Therefore, we have to strictly monitor RE to reduce the exposure down to the lowest level needed to allow the procedure to be completed in a safe and timely manner, following the 'as low as reasonably achievable' (ALARA) principle [15-17]. To date, there are few reports about the actual RE of FGPs. Unfortunately, there are still no available national DRLs in gastrointestinal endoscopy departments, and there are very few local DRLs. Therefore, we examined the actual RE of FGPs in the present study in order to set local DRLs in our endoscopy unit.

\section{Patients and methods}

This was a retrospective, single-center cohort study of consecutive patients who underwent FGPs, including ERCP, EUS cyst drainage (EUS-CD), self-expandable enteral stenting (SEMS), balloon-assisted enteroscopy (BAE), esophageal balloon dilatation (esophageal BD), endoscopic injection sclerotherapy (EIS), tube placement and repositioning for sigmoid volvulus (Rep SV) under the over- tube fluoroscopy device at the Toyonaka Municipal Hospital, which was certified as a teaching hospital by the Japan Gastroenterological Endoscopy Society (JGES) (No.1239) and the Japanese Society of Gastroenterology (JSGE) (No. 27020). The study period was between September 2012 and June 2019. We have two fluoroscopy devices in the endoscopy department. All FGPs were performed with the same fluoroscopy device, EXAVISTA 17 (Hitachi Co., Japan). We had two ima- ging processing units for the EXAVISTA system (FAiCE-V, Hitachi.co, Japan). In the middle of the study period, we updated one of these devices: the new image processing engine, FAiCEV Next Stage 1 (NS1), has been available since July 2016. During the study period, we consecutively examined the air kerma (AK: $\mathrm{mGy}$ ) and dose area product (DAP: $\mathrm{Gycm}^{2}$ ) as the radiation dose and fluoroscopy time (FT: $\mathrm{min}$ ) of each procedure. The IAEA recommends DAP for fluoroscopy and interventional radiology procedures as the primary DRL quantity. The AK and FT are recommended as useful additional DRL quantities. Therefore, we adapted the DAP, AK, and FT as DRL quantities in the present study [5]. The AK, DAP and FT of each procedure were automatically calculated using the numerical dose determination method of the fluoroscopy unit. National and regional DRLs are usually set at the $75 \%$ percentile of the distribution of a typical sample dose [18]. According to the DRL concept, we expressed the median and third quartile values of the radiation dose. The present study was conducted in accordance with the Declaration of Helsinki, and approval was obtained from the institutional review board.

\section{Definition of factors}

The FT and radiation dose were automatically recorded for each procedure by the fluoroscopy device. The procedures were divided into the following eight categories: 1) ERCP, which is used for common bile duct stones (CBDSs), distal malignant bile duct obstruction (DMBO), proximal malignant bile duct obstruction (PMBO) and others; 2) SEMS, which includes esophageal SEMS (ES), gastroduodenal SEMS (GDS) and colorectal SEMS (CRS); 3) tube placement, which includes elemental diet tube (ED tube), transnasal ileus tube (nasal tube) and transanal ileus tube (rectal tube); 4) balloon-assisted enteroscopy; 5) EUS-CD; 6) EIS; 7) esophageal balloon dilatation; and 8) repositioning for sigmoid volvulus.

\section{Statistical analysis}

All of the continuous variables are expressed as the median and third quartile. The categorical variables are expressed as the number or frequency. All statistical analyses were performed with the use of JMP software (ver. 14.0.0, SAS Institute Inc., Cary, North Carolina, United States).

\section{Results \\ Inclusion tree and patient characteristics}

A total of 4865 FGPs were performed. Among them, 1034 procedures that were not interventions, such as contrast studies, removal of a foreign body or drainage tube, intubation assistance, and those with missing data, were excluded. Finally, a total of 3831 FGPs were analyzed in the present study ( $\triangleright$ Fig. 1). The mean age was $73.4 \pm 12.1$ years, and 1556 patients were female (40.6\%). The procedures included ERCP $(n=2778$, $57.1 \%)$, EUS-CD ( $n=14,0.3 \%)$, SEMS ( $n=216,4.4 \%)$, balloonassisted enteroscopy ( $n=30,0.6 \%)$, esophageal balloon dilatation $(n=136,2.8 \%)$, EIS $(n=60,1.2 \%)$, tube placement $(n=$ $536,11.0 \%$ ), repositioning for sigmoid volvulus ( $n=59,1.2 \%)$, 


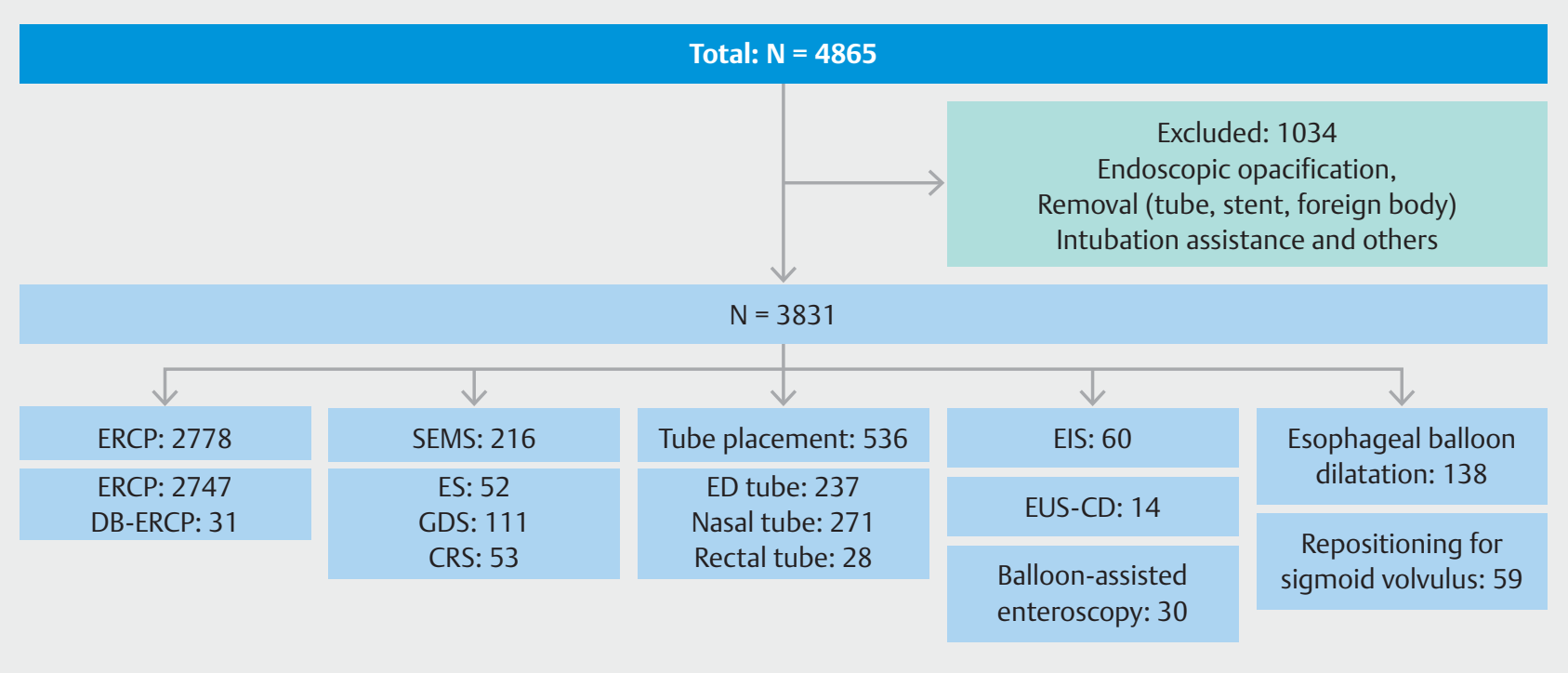

> Fig. 1 Study flowchart. ERCP, endoscopic retrograde cholangiopancreatography; DB-ERCP, double balloon-assisted ERCP; SEMS, self-expandable metallic stent; ES, esophageal SEMS; GDS, gastroduodenal SEMS; CRS, colorectal SEMS; ED tube, elemental diet tube; EIS, endoscopic injection sclerotherapy; EUS-CD, endoscopic ultrasound-guided cyst drainage.

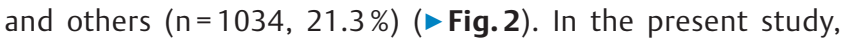
ERCP and tube placement were the most common procedures among FGPs.

\section{AK, DAP and FT of each procedure}

For ERCP, the median/third quartile values of AK (mGy), DAP $\left(G_{y c m}^{2}\right)$ and FT (min) were 109/234 mGy, 13.3/25.8 Gycm ${ }^{2}$ and $10.0 / 16.0 \mathrm{~min}$. Similarly, these values were $62 / 101 \mathrm{mGy}$, $12.4 / 18.5 \mathrm{Gycm}^{2}$ and $10.4 / 14.2 \mathrm{~min}$ for SEMS, 40/71 mGy, $13.8 / 25.5 \mathrm{Gycm}^{2}$ and $11.1 / 16.2 \mathrm{~min}$ for tube placement, 43/ $70 \mathrm{mGy}, 22.4 / 40.6 \mathrm{Gycm}^{2}$ and 18.2/27.7 min for balloon-assisted enteroscopy, 96/207 mGy, 18.3/24.1 Gycm ${ }^{2}$ and 10.4/ $13.1 \mathrm{~min}$ for EUS-CD, 36/57 mGy, 8.1/13.1 Gycm ${ }^{2}$ and 4.4/5.5 min for EIS, 9/16 mGy, 2.2/4.3 Gycm ${ }^{2}$ and $1.8 / 3.6 \mathrm{~min}$ for esophageal balloon dilatation, and 7/13 mGy, 4.7/10.3 Gycm² and 1.6/3.9 min for repositioning for sigmoid volvulus, respectively. $>$ Table 1 shows the details of each procedure.

\section{Discussion}

The trend of increasing medical RE is a significant concern throughout society because of its potential cancer risk $[2,3,19]$. RE has two significant adverse effects. One is the deterministic effect, which has the potential to induce tissue injury such as skin injury, ulcers or cataracts. The other is the stochastic effect, in which linear increases in low dose lead to gene damage and ultimately cancer [20,21]. Medical RE was estimated to account for approximately $48 \%$ of the effective dose to the US population in 2006, compared to the $15 \%$ in the early 1980 s, according to the National Council on Radiation Protection and Measurements (NCRP) report no. 160 [22-24]. Of the medical procedures, computed tomography (CT) accounted for approximately half of the medical RE, followed by fluoroscopic

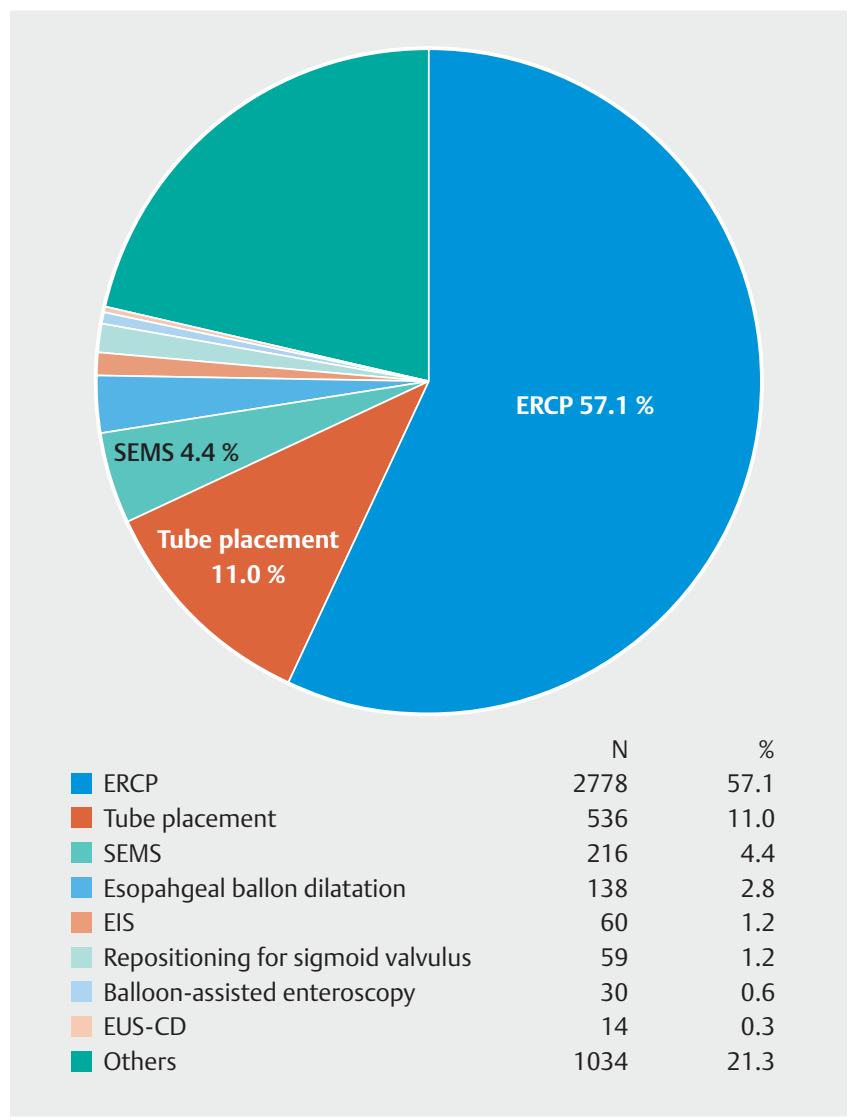

Fig. 2 Proportion of FGPs. ERCP, endoscopic retrograde cholangiopancreatography; SEMS, self-expandable metallic stent; EIS, endoscopic injection sclerotherapy; EUS-CD, endoscopic ultrasound-guided cyst drainage. 
- Table 1 Median and third-quartile values of AK, DAP, and FT for each procedure.

\begin{tabular}{|c|c|c|c|c|}
\hline Procedure & $\mathbf{N}$ & $\begin{array}{l}\text { AK (mGy) } \\
\text { Median, } 3^{\text {rd }} \text { quartile }\end{array}$ & $\begin{array}{l}\text { DAP }\left(G y c^{2}\right) \\
\text { Median, } 3^{\text {rd }} \text { quartile }\end{array}$ & $\begin{array}{l}\text { FT (min) } \\
\text { Median, } 3^{\text {rd }} \text { quartile }\end{array}$ \\
\hline ERCP & 2778 & 109,234 & $13.3,25.8$ & $10.0,16.0$ \\
\hline CBDS & 1325 & 120,248 & $13.7,27.6$ & $10.1,16.6$ \\
\hline DMBO & 480 & 85,182 & $11.1,20.4$ & $8.1,13.0$ \\
\hline PMBO & 360 & 145,298 & $17.0,32.6$ & $13.2,20.0$ \\
\hline DB-ERCP & 31 & 68,101 & $12.8,17.5$ & $20.8,29.8$ \\
\hline SEMS & 216 & 62,101 & $12.4,18.5$ & $10.4,14.2$ \\
\hline ES & 52 & 45,69 & $6.9,11.8$ & $6.4,9.9$ \\
\hline GDS & 111 & 75,122 & $14.3,19.5$ & $11.8,16.7$ \\
\hline CRS & 53 & 58,106 & $14.9,21.9$ & $10.7,14.1$ \\
\hline Tube placement & 536 & 40,71 & $13.8,25.5$ & $11.1,16.2$ \\
\hline ED tube & 237 & 32,51 & $10.8,19.7$ & $6.3,12.2$ \\
\hline Nasal tube & 271 & 53,82 & $17.8,27.7$ & $12.5,18.5$ \\
\hline Rectal tube & 28 & 49,108 & $15.4,26.2$ & $7.1,12.7$ \\
\hline Balloon-assisted enteroscopy & 30 & 43,70 & $22.4,40.6$ & $18.2,27.7$ \\
\hline EUS-CD & 14 & 96,207 & $18.3,24.1$ & $10.4,13.1$ \\
\hline EIS & 60 & 36,57 & $8.1,13.1$ & $4.4,5.5$ \\
\hline Esophageal balloon dilatation & 138 & 9,16 & $2.2,4.3$ & $1.8,3.6$ \\
\hline Repositioning for sigmoid volvulus & 59 & 7,13 & $4.7,10.3$ & $1.6,3.9$ \\
\hline
\end{tabular}

procedures and nuclear medicine, which accounted for approximately one-quarter of the medical RE. Therefore, the radiation used in medical imaging, including fluoroscopic procedures, must be measured and managed [25]. To adequately manage medical RE, the ICRP and IAEA introduced DRLs, in which the actual value of each procedure is usually set at the third quartile value (75th percentile) of a large sample size to indicate whether the patient dose and administered activity from a specified procedure are unusually high or low [26]. Almost all diagnostic procedures have previously set DRLs that have been updated for several years, but not enough interventional procedures have yet received DRLs. Nationwide DRLs for endoscopic procedures performed under fluoroscopy are still not well established $[27,28]$. Currently, there is still not enough available data on RE from endoscopic FGPs, although the European Commission, UK, and German DRL set a single value for ERCP [29-31. The present report of third quartile values of FGPs, which is the first of its kind, will serve as a useful reference $(\triangleright$ Table 1$)$.

Little data on RE in the endoscopy department are available because interventional procedures are complex, as described in the ICRP supporting guidance 2 [32]. This complexity means that each intervention has different goals and difficulties.
Therefore, a single DRL value is insufficient to cover the interventions, and several values are recommended for guidance. The German DRLs attempted to set three DRL values for interventional procedures, such as those to treat aneurysm and angioplasty [31]. In the gastrointestinal field, Schmitz et al. reported multicenter data on percutaneous biliary interventions in Germany, covering 564 procedures classified into 8 types [33]. We have reported a simple disease site classification of $E R C P$, which includes treatment focused on three sites: proximal malignant biliary obstruction (PMBO), distal MBO and common bile duct stones (CBDSs) [34]. In this study, ERCP was the most popular FGP $(n=2778,57.1 \%)$, and it might be reasonable to introduce such a simple classification for ERCP. In this way, there have been attempts to establish DRLs for IVR procedures, where grouping by disease site may help minimize the wide distribution of RE [34, 35].

DRL quantities are not actually equal to the quantities that patients receive. Those values have a different definition and are difficult to compare directly. Among radiation dose quantity data, the effective dose is a suitable value with which to compare radiation dose levels of different imaging modalities and is used to assess the potential for long-term cancer risk. We estimated the effective dose from DAP data using the dose conver- 
sion coefficient (biliary: 0.26 ) reported by Li et al. [36], which showed that the median and 3 rd quartile DAP values of ERCP were comparable to $3.5 / 6.7 \mathrm{mSv}$. CT is the most popular and important in type of medical procedure involving radiation. The effective dose of plain abdominal CT is approximately 10 $\mathrm{mSv}$ [37]. The tentative median effective dose of ERCP is approximately one-third of the dose of plain abdominal CT. However, it should be noted that the radiation exposure of CT has low variance, but that of FGPs has a wide range.

There are several limitations to the current study. First, this study was a single-center retrospective study. Gastrointestinal fluoroscopic procedures have been rapidly increasing in number and complexity. However, there are still no available worldwide or national DRLs in gastrointestinal endoscopy departments, and few local DRLs have been established. The ICRP 135 recommends that a survey for a particular examination in a facility should normally involve the collection of data on the local DRL quantity for at least 20 patients [38]. In the present study, we planned to set local DRLs at our endoscopy unit, and we collected radiation dose data for more than 20 procedures, including 8 FGPs, which was sufficient to establish local DRLs for all procedures except EUS-CD. The second limitation involved local DRLs, which can be strongly affected by the conditions of a facility. In particular, updating the fluoroscopy device could have strongly affected the radiation dose in the present study [39]

\section{Conclusion}

In conclusion, we were the first to show actual RE data for the following interventional procedures in gastrointestinal endoscopy departments: traditional or double balloon-assisted ERCP, interventional EUS, balloon-assisted enteroscopy, enteral metallic stent placement, and enteral tube placement. We are prospectively conducting the REX-GI study (UMIN000036525) to collect actual RE data to establish national DRLs for the interventional procedures performed in gastrointestinal endoscopy departments [40].

\section{Competing interests}

The authors declare that they have no conflict of interest.

\section{References}

[1] Balter S, Miller DL. Patient skin reactions from interventional fluoroscopy procedures. Am J Roentgenol 2014; 202: W335-W342

[2] de González AB, Darby S. Risk of cancer from diagnostic X-rays: estimates for the UK and 14 other countries. Lancet 2004; 363: 345-351

[3] Mathews JD, Forsythe AV, Brady Z et al. Cancer risk in 680,000 people exposed to computed tomography scans in childhood or adolescence: data linkage study of 11 million Australians. BM] 2013; 346: $\mathrm{f} 2360$

[4] ICRP. Avoidance of radiation injuries from medical interventional procedures, ICRP publication 85. Ann ICRP 2000; 30: 7-67. doi:10.1016/S0146-6453(01)00004-5
[5] International Atomic Energy Agency. Diagnostic Reference Levels (DRLs). Available at (Accessed Sep 2, 2020): https://www.iaea.org/ resources/rpop/health-professionals/radiology/diagnostic-referencelevels

[6] Japan Network for Research and Information on Medical Exposure. Diagnostic Reference Levels Based on Latest Surveys in Japan - Japan DRLs 2015 -. Available at (Accessed Sep 2, 2020): http://www.radher.jp/J-RIME/report/DRLhoukokusyoEng.pdf

[7] Watanabe $\mathrm{H}$, Ishii K, Hosono $\mathrm{M}$ et al. Report of a nationwide survey on actual administered radioactivities of radiopharmaceuticals for diagnostic reference levels in Japan. Ann Nucl Med 2016; 30: 435-444

[8] Miller DL, Vano E, Bartal G et al. Occupational radiation protection in interventional radiology: a joint guideline of the Cardiovascular and Interventional Radiology Society of Europe and the Society of Interventional Radiology. Cardiovasc Intervent Radiol 2010; 33: 230-239

[9] Miller DL. Overview of contemporary interventional fluoroscopy procedures. Health Phys 2008; 95: 638-644

[10] Huang RJ, Thosani NC, Barakat MT et al. Evolution in the utilization of biliary interventions in the United States: results of a nationwide longitudinal study from 1998 to 2013. Gastrointest Endosc 2017; 86: 319-326 e315

[11] Adler DG, Lieb JG 2nd, Cohen J et al. Quality indicators for ERCP. Gas trointest Endosc 2015; 81: 54-66

[12] Dumonceau JM, Garcia-Fernandez F], Verdun FR et al. Radiation protection in digestive endoscopy: European Society of Digestive Endoscopy (ESGE) guideline. Endoscopy 2012; 44: 408-421

[13] ICRP. ICRP publication 118: ICRP statement on tissue reactions and early and late effects of radiation in normal tissues and organsthreshold doses for tissue reactions in a radiation protection context. Ann ICRP 2012; 41: 1-322

[14] Stecker MS, Balter S, Towbin RB et al. Guidelines for patient radiation dose management. J Vasc Interv Radiol 2009; 20: S263-273

[15] Winkler NT. ALARA concept-now a requirement. Radiol Technol 1980; 51: 525

[16] Hendee WR, Edwards FM. ALARA and an integrated approach to radiation protection. Semin Nucl Med 1986; 16: 142-150

[17] Brateman L. Radiation safety considerations for diagnostic radiology personnel. Radiographics 1999; 19: 1037-1055

[18] Vassileva J, Rehani M. Diagnostic reference levels. AJR Am J Roentgenol 2015; 204: W1-3

[19] Jaffe D, Bowden GT. lonizing radiation as an initiator: effects of proliferation and promotion time on tumor incidence in mice. Cancer Res 1987; 47: 6692-6696

[20] Cardis E, Gilbert E, Carpenter L et al. Effects of low doses and low dose rates of external ionizing radiation: cancer mortality among nucle. Radiat Res 1995; 142: 117-132

[21] Richardson DB, Cardis E, Daniels RD et al. Risk of cancer from occupational exposure to ionising radiation: retrospective cohort study of workers in France, the United Kingdom, and the United States (INWORKS). BM] 2015; 351: h5359

[22] Schauer D, Linton O. National council on Radiation Protection and Measurements Report Shows Substantial Medical Exposure Increase. Radiology 2009; 253: 293-296

[23] Bolus NE. NCRP report 160 and what it means for medical imaging and nuclear medicine. J Nucl Med Technol 2013; 41: 255-260

[24] NCRP. Ionizing radiation exposure of the population of the United States. National Council on Radiation Protection report no. $160 \mathrm{Na}-$ tional Council on Radiation Protection and Measurements. Bethesda, Md: National Council on Radiation Protection and Measurements; 2009

[25] Hricak H, Brenner D, Adelstein $\mathrm{S}$ et al. Managing radiation use in medical imaging: a multifaceted challenge. Radiology 2011; 258: 889-905 
[26] ICRP. The 2007 Recommendations of the International Commission on Radiological Protection. ICRP publication 103. Ann ICRP 2007; 37: $1-332$

[27] Hayashi S, Takenaka M, Hosono M et al. Radiation exposure during image-guided endoscopic procedures: The next quality indicator for endoscopic retrograde cholangiopancreatography. World J Clin Cases 2018; 6: 1087-1093

[28] Saukko E, Henner A, Nieminen MT et al. The establishment of local diagnostic reference levels in endoscopic retrograde cholangiopancreatography: a practical tool for the optimisation and for quality assurance management. Radiat Prot Dosimetry 2017; 173: 338-344

[29] European Commission. Diagnostic Reference Levels in Thirty-six European Countries. Available at (Accessed 2020 Sep 2): https://ec. europa.eu/energy/sites/ener/files/documents/RP180\%20part2.pdf

[30] Government of United Kingdom. National Diagnostic Reference Levels (NDRLs) from 19 August 2019. Available at (Accessed 2020 Sep 2): https://www.gov.uk/government/publications/diagnostic-radiologynational-diagnostic-reference-levels-ndrls/ndrl

[31] Bundesamt fur Strahlenschutz. Bekanntmachung der aktualisierten diagnostischen Referenzwerte für diagnostische und interventionelle Röntgenanwendungen. 2016; 22

[32] ICRP. Radiation and your patient - A Guide for Medical Practitioners. ICRP Supporting Guidance 2. Ann ICRP 2001; 31: 5-31 (PMID: 12685757)

[33] Schmitz D, Vogl T, Nour-Eldin NA et al. Patient radiation dose in percutaneous biliary interventions: recommendations for DRLs on the basis of a multicentre study. Eur Radiol 2019; 29: 3390-3400
[34] Hayashi S, Nishida T, Matsubara T et al. Radiation exposure dose and influencing factors during endoscopic retrograde cholangiopancreatography. PLoS One 2018; 13: e0207539

[35] Bundesamt fur Strahlenschutz. Bekanntmachung der aktualisierten diagnostischen Referenzwerte für diagnostische und interventionelle Röntgenanwendungen Vom 22. Juni 2016. Available at (Accessed 2020 Sep 2): https://www.bfs.de/SharedDocs/Downloads/BfS/DE/fachinfo/ion/drw-roentgen.pdf?__blob=publicationFile\&v=9

[36] Li X, Hirsch JA, Rehani MM et al. Effective dose assessment for patients undergoing contemporary fluoroscopically guided interventional procedures. AJR Am J Roentgenol 2020; 214: 158-170

[37] Radiological Society of North America. Radiation Dose in X-Ray and CT Exams. 2020: Available at (Accessed 2020 Sep 2): https://www. radiologyinfo.org/en/info.cfm?pg=safety-xray

[38] Vano E, Miller DL, Martin CJ et al. ICRP Publication 135: Diagnostic Reference Levels in Medical Imaging. Ann ICRP 2017; 46: 1-144

[39] Hayashi S, Nishida T, Osugi N et al. Time trend of the radiation exposure dose in endoscopic retrograde cholangiopancreatography over an 8-year period: a single-center retrospective study. Am J Gastroenterol 14.09 2020: doi:10.14309/ajg.0000000000000838

[40] Nishida T, Hayashi S, Takenaka M et al. Multicentre prospective observational study protocol for radiation exposure from gastrointestinal fluoroscopic procedures (REX-GI study). BMJ Open 2020; 10: e033604doi:10.1136/bmjopen-2019-033604 